\title{
Photo- and Thermal-Stability Studies on Benzimidazole Anthelmintics by HPLC and GC-MS
}

\author{
Gaetano Ragno,* Antonella Risoli, Giuseppina Ioele, and Michele De Luca \\ University of Calabria, Pharmacy Faculty, Department of Pharmaceutical Sciences; 87036 Rende (CS), Italy. \\ Received November 21, 2005; accepted March 15, 2006
}

\begin{abstract}
Photo- and thermal-stability of the anthelmintics Albendazole, Mebendazole and Fenbendazole as in solid as in solution form has been investigated, by using a Xenon arc lamp as a radiation source, according to the ICH guideline for the drug stability tests. The degradation process was monitored by a HPLC method. All drugs showed high photosensitivity in solution but a reliable stability in solid form and when exposed to a temperature up to $50^{\circ} \mathrm{C}$. Two main degradation products from hydrolysis of the carbamic groups were identified by GC-MS. Validation studies demonstrated high accuracy (recovery 94 to 106\%) and precision (RSD under $4.6 \%$ ) of the HPLC method. The analytical procedure was successfully applied to the control of the drugs in the respective pharmaceutical formulations.
\end{abstract}

Key words benzimidazole anthelmintics; photodegradation; thermal-degradation; HPLC; GC-MS

Infections by worms represent a serious health problem in the tropical and subtropical regions, when the environmental conditions are especially critical for the drug storage, due to both high temperature and strong sunlight. Under these stressing climatic conditions, the characterization of the thermal- and photo-stability of drugs and drug formulations is of high interest. Light and high temperature can influence a drug formulation, leading to changes in the physico-chemical and therapeutic properties of the active principles. ${ }^{1-4)}$

The most common drugs used as anthelmintics belong to the benzimidazole series of which the most commercialized in the world are: Albendazole (ALB), Fenbendazole (FEN) and Mebendazole (MEB) ${ }^{5-12)}$ (Fig. 1). Benzimidazole drugs inhibit in an irreversible way the glucose uptake leading to the depletion of glycogen storage of the parasites. ${ }^{13,14)}$

Due to their chemical features, all the benzimidazole drugs are sensitive to light, with a behaviour common to all members of this class of compounds. ${ }^{13,15)}$ The amine derivative from hydrolysis of the carbamic group has been reported to be the main photodegradation product, indicated also by metabolism studies as the major metabolite of the drugs. ${ }^{13)}$ Accordingly, a close monitoring of these drugs, as such or in pharmaceutical formulations, constitutes a noteworthy analytical problem in regions presenting tropical climate. HPLC procedures have been used for determination of $\mathrm{MEB},{ }^{15}$ ) $\mathrm{ALB}^{16,17)}$ and $\mathrm{FEN}^{17}$ in veterinary formulations whereas only a TLC method for the degradation study of MEB is reported. ${ }^{11)}$

In the present paper, the studied drugs were exposed to various conditions of stressing light and temperature. The degradation study was performed under controlled condi-

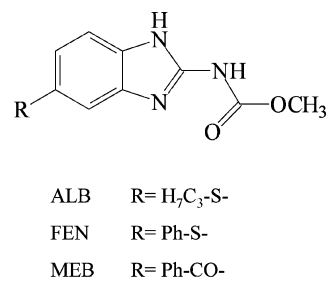

Fig. 1. Chemical Structure of Albendazole, Fenbendazole and Mebendazole

* To whom correspondence should be addressed. e-mail: ragno@unical.it tions, using a cabinet provided with a Xenon lamp, able to simulate the solar light spectrum, and a temperature controller.

The photoproducts were monitored by HPLC and characterized by GC-MS. HPLC method was validated by analysis of synthetic mixtures containing different amounts of drugs and photoproducts. HPLC method was finally applied to the analytical control of the drugs commercial formulations.

\section{Results and Discussion}

Photo-Degradation Study Ethanol solutions of ALB, FEN and MEB $(20 \mathrm{mg} / \mathrm{ml})$ were exposed to controlled irradiation and analyzed at time intervals up to $10 \mathrm{~h}$. Figure 2 shows semi-logarithmic plots of the \% residue drug $v s$. irradiation time. The concentration of the products were obtained as a function of the respective peak areas carried out by HPLC chromatography. The drugs concentration values were carried out by using the relationships reported in Table 1. The exposure to light of the drugs in ethanol solution caused a marked degradation for all the compounds.

Figure 3 shows the chromatograms of the ethanol solutions

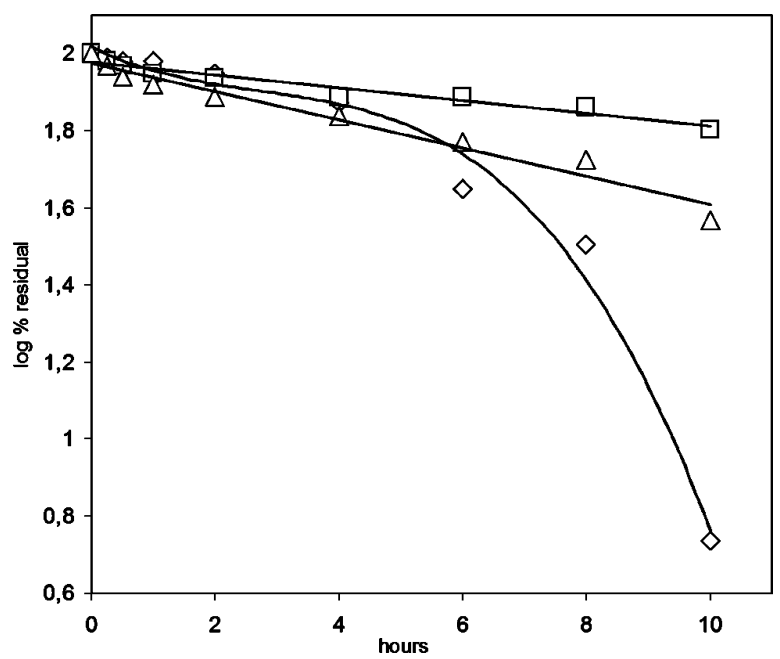

Fig. 2. Photodegradation Curves of ALB $(\diamond), \operatorname{FEN}(\square)$ and $\operatorname{MEB}(\triangle)$ in Ethanol Solutions 
Table 1. Linearity Relationships for Benzimidazole Drugs and Related Photoproducts

\begin{tabular}{|c|c|c|c|c|}
\hline Analyte & Retention time & Slope & Intercept & $r^{2}$ \\
\hline ALB & 4.93 & $14121.4( \pm 393.4)$ & $-291.4( \pm 195.3)$ & 0.9907 \\
\hline ALB-DEG1 & 0.86 & $12041.7( \pm 298.9)$ & $158.0( \pm 96.5)$ & 0.9983 \\
\hline ALB-DEG2 & 1.72 & $13529.5( \pm 370.5)$ & $521.4( \pm 285.3)$ & 0.9756 \\
\hline FEN & 7.46 & $15405.1( \pm 405.7)$ & $317.9( \pm 207.8)$ & 0.9923 \\
\hline FEN-DEG1 & 0.84 & $13874.1( \pm 387.4)$ & $210.3( \pm 85.1)$ & 0.9888 \\
\hline FEN-DEG2 & 1.84 & $13048.7( \pm 365.2)$ & $265.9( \pm 137.0)$ & 0.9936 \\
\hline MEB & 3.14 & $19770.3( \pm 582.3)$ & $-407.9( \pm 219.1)$ & 0.9855 \\
\hline MEB-DEG1 & 1.44 & $15718.6( \pm 0.473)$ & $236.7( \pm 173.3)$ & 0.9621 \\
\hline MEB-DEG2 & 2.34 & $16026.0( \pm 0.557)$ & $510.3( \pm 228.2)$ & 0.9421 \\
\hline
\end{tabular}

In parentheses are reported the confidence intervals; significance level, $p=0.05$.

$(20 \mathrm{mg} / \mathrm{ml})$ for the drugs after an irradiation time of $4 \mathrm{~h}$, corresponding to about $3800 \mathrm{~kJ} / \mathrm{m}^{2}$ of irradiance. A similar behaviour in the degradation process was observed for all the drugs. The clear formation of a peak (DEG1), with a low retention time, was pointed out at $0.86,0.84$ and $1.44 \mathrm{~min}$ for ALB, FEN and MEB, respectively. The retention times for the peaks of the intact drugs were $4.93 \mathrm{~min}$ for ALB, $7.46 \mathrm{~min}$ for FEN and $3.14 \mathrm{~min}$ for MEB. A further irradiation caused the formation of a second peak (DEG2) with retention times at 1.72, 1.84 and $2.34 \mathrm{~min}$ for ALB, FEN and MEB, respectively.

The HPLC study revealed the formation of a large number of minor photoproducts in the photodegradation process of MEB, whereas ALB degradation presented a higher rate compared with the other two drugs. The plots for FEN and MEB showed good linearity ( $r>0.997)$, suggesting that the photodegradation followed apparently a first-order kinetics. On the contrary, the degradation profile for ALB did not show linearity and the degradation rate increased exponentially with time. Really, the photodegradation test for FEN and MEB showed a slow but constant increase of both photoproducts DEG1 and DEG2, nevertheless their concentration never reached a concentration over the $30 \%$ of the initial drug amount. On the contrary, for ALB samples the photoproduct DEG2 increased up to reach a percentage concentration of $80 \%$ of the starting drug while a simultaneous quite slowdown of DEG1 was observed. The photodegradation process of the benzimidazole drugs therefore seemed to involve at least two consecutive steps in which a first degradation product DEG1 was subsequently decomposed to degradation product DEG2 and then other substances further along the decomposition chain.

The degradation compounds DEGs1-2 were isolated and analyzed by GC-MS. The photoproducts DEGs1 showed a molar mass with $\mathrm{m} / \mathrm{z}$ corresponding to the related demethylated products of the parent drugs, suggesting a demethylation as the first step of the degradation process. The main mass fragments resulted $\mathrm{m} / \mathrm{z}: 251\left(\mathrm{M}^{+}-\mathrm{CH}_{3}\right) ; 207$ $\left(\mathrm{M}^{+}-\mathrm{COOCH}_{3}\right)$ for ALB-DEG1 and $m / z: 285\left(\mathrm{M}^{+}-\mathrm{CH}_{3}\right)$; $241\left(\mathrm{M}^{+}-\mathrm{COOCH}_{3}\right)$ for FEN-DEG1. The MEB photoproduct DEG1 was isolated in a low amount and not strictly purified. Anyhow, its structure was confirmed by GC-MS, presenting a molecular ion with $m / z$ value $281\left(\mathrm{M}^{+}-\mathrm{CH}_{3}\right)$ and a fragment $m / z 237\left(\mathrm{M}^{+}-\mathrm{COOCH}_{3}\right)$.

The photoproducts DEGs2 showed mass spectra in agreement with the literature reports, ${ }^{15)}$ corresponding to the amine DEGs-1 by-products from the decarboxylation of the carbamic groups. The formation of this product was particularly evident for ALB in respect to the other two drugs. The scheme of Fig. 4 shows the supposed overall reaction involved in the photodegradation of the benzimidazole drugs.

Effectively, the formation of two photoproducts in the MEB photodegradation process has been cited by El Fatih (1996), but no chemical structure has been reported. Moreover, the formation of the de-methylated degradation product is not mentioned at all, indicating the 2-amino-benzimidazole derivative as the main degradation product. ${ }^{15)}$ Since this is the first time that the de-methylated photoproducts are isolated, a consequent investigation about the residual pharmacological activity of them should be performed in the next future.

In contrast with the clear photodegradation process in solution form, the direct exposure to light of the drugs in solid form was demonstrated to cause no appreciable changes. The absence of degradation in the solid form could be explained with the need of water to support the hydrolysis process. The high stability presented by the solid form of the drugs confirms the advisability to manufacture these anthelmintic drugs just in tablets or similar solid pharmaceutical forms.

Thermal-Degradation Study The thermal degradation test, performed under temperatures from 20 to $50^{\circ} \mathrm{C}$, demonstrated a very high stability both in solid and solution form, as shown in Fig. 5. These temperature values were considered a reliable range that can be reached in the drug storages of the tropical lands.

These results confirmed that the degradation process was caused just by light and not by high temperature. In support of this thesis, a further degradation test was performed by subjecting the ethanol solutions of drugs to both stressing conditions, exposing them in the light cabinet under an irradiation intensity of $21 \mathrm{~kJ} / \mathrm{min} / \mathrm{m}^{2}$ and temperatures up to $50{ }^{\circ} \mathrm{C}$. The degradation profiles of the samples resulted practically superimposable with those carried out from the simple light irradiation.

The defined HPLC method could be used for the simultaneous determination of the drugs and their photodegradation products in pharmaceutical formulations as well as in bulk. It was applied to the analytical control of the commercial pharmaceuticals of the benzimidazole anthelmintics studied. The assay results, shown in Table 2, were in satisfactory accordance with the drug declared amounts. The photoproducts were demonstrated to be not more than $2.5 \%$.

Validation of the HPLC Method Linearity of the HPLC method was checked by analyzing five standard solu- 

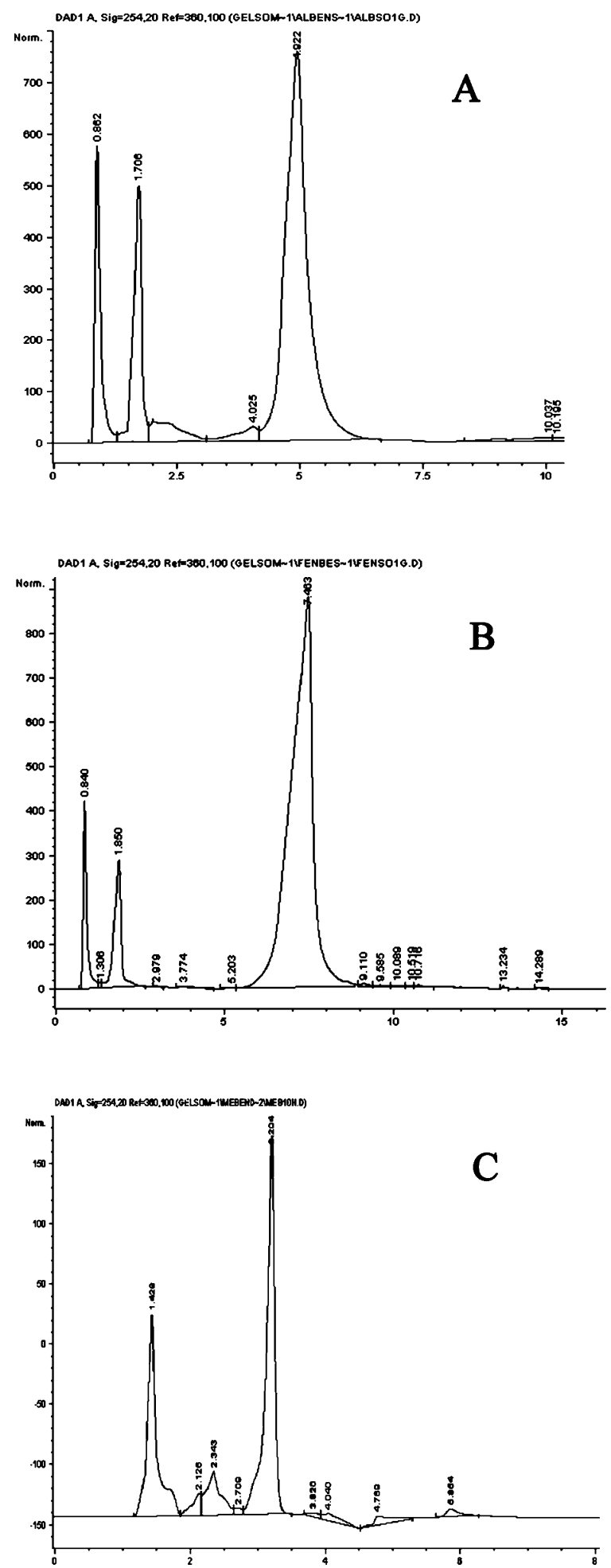

Fig. 3. HPLC Chromatograms of Benzimidazole Drugs and Related Photoproducts

(A) ALB $4.93 \mathrm{~min}$, ALB-DEG1 $0.86 \mathrm{~min}$, ALB-DEG2 $1.72 \mathrm{~min}$; (B) FEN $7.46 \mathrm{~min}$, FEN-DEG1 $0.84 \mathrm{~min}$, FEN-DEG2 $1.84 \mathrm{~min}$; (C) MEB $3.14 \mathrm{~min}$, MEB-DEG1 $1.44 \mathrm{~min}$, MEB-DEG2 $2.34 \mathrm{~min}$

tions for each product, with concentration ranging from 0.1 to $2.0 \mathrm{mg} / \mathrm{ml}$ for pure drugs and 0.01 to $0.20 \mathrm{mg} / \mathrm{ml}$ for degradation products. Relationships between products concentration versus relative peak areas were carried out by linear regression analysis and summarized in Table 1.

Detection limit of each photoproduct was determined by

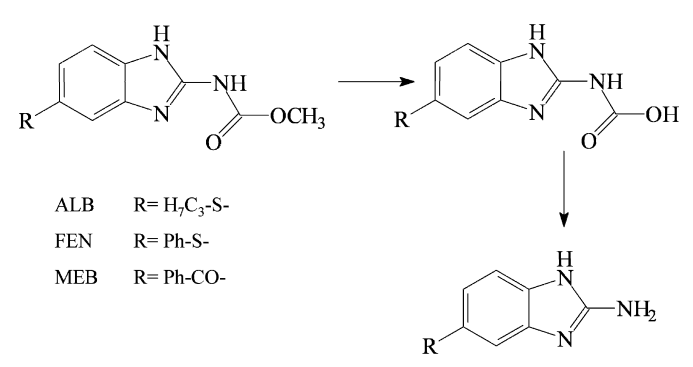

Fig. 4. Photodegradation Pattern of Benzimidazole Anthelmintics

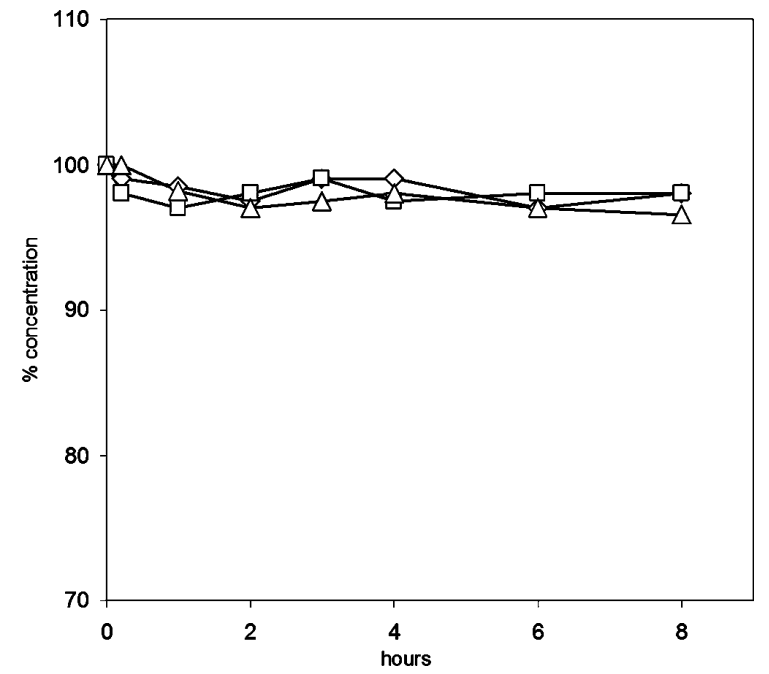

Fig. 5. Thermal-Degradation Curves of $\operatorname{ALB}(\diamond), \operatorname{FEN}(\square)$ and $\operatorname{MEB}(\triangle)$ in Ethanolic Solutions

diluting a standard solution until the average HPLC response resulted approximately three times the standard deviation of the response from a $0.1 \mathrm{mg} / \mathrm{ml}$ standard solution. The quantitation limit was determined as the concentration value giving an average HPLC response approximately ten times the standard deviation of the response from a $0.1 \mathrm{mg} / \mathrm{ml}$ standard solution. The detection and quantitation limits for all the photoproducts resulted less than or equal to 0.005 and $0.015 \mathrm{mg} / \mathrm{ml}$, respectively.

Accuracy and precision of the HPLC method were evaluated by analysis of triplicate samples containing standard mixtures of drugs and related photodegradation products with concentration within the ranges described above. Accuracy values, expressed as recovery $\%$, were calculated to be in the range $96-104 \%$ and $94-106 \%$, whereas precision values, expressed as RSD\%, were calculated within the range $1.3-3.6 \%$ and $1.6-4.6 \%$ for parent drugs and degradation products, respectively. The statistical data are summarized in Table 2. The robustness of the HPLC method was also checked, in agreement with the ICH definition, ${ }^{18)}$ measuring its capacity to remain unaffected by small variations in method parameters. For this purpose, the mean instrumental parameters, temperature and mobile phase composition, were demonstrated to cause negligible variation of the chromatographic response when their values were varied within the limits of $5 \%$.

\section{Experimental}

Instruments HPLC assays were carried out using a HP 1100 Pump fitted with a diode array detector 2K-2280 (Agilent Technologies) and a Rheo- 
Table 2. Accuracy and Precision Data from HPLC Assay of Benzimidazole Drugs and Related Photodegradation Products (DEG) in Synthetic Mixtures and Commercial Formulations

\begin{tabular}{|c|c|c|c|c|c|c|}
\hline \multirow{2}{*}{ Sample } & \multicolumn{3}{|c|}{ Nominal } & \multicolumn{3}{|c|}{ Found (RSD\%) } \\
\hline & Drug & DEG1 & DEG2 & Drug & DEG1 & DEG2 \\
\hline \multicolumn{7}{|l|}{ Albendazole } \\
\hline Sample 1 & 0.202 & 0.050 & 0.103 & $0.194(2.02)$ & $0.052(3.48)$ & $0.098(3.07)$ \\
\hline Sample 2 & 1.012 & 0.074 & 0.309 & $0.982(3.56)$ & $0.076(2.89)$ & $0.328(3.85)$ \\
\hline Sample 3 & 2.024 & 0.148 & 1.236 & $2.105(1.34)$ & $0.144(4.58)$ & $1.187(2.43)$ \\
\hline Zentel $^{\circledR}$ & 400 & - & - & $385.440(3.20)$ & $5.521(1.59)$ & $4.254(4.52)$ \\
\hline \multicolumn{7}{|l|}{ Fenbendazole } \\
\hline Sample 1 & 0.480 & 0.031 & 0.080 & $0.490(1.80)$ & $0.032(3.32)$ & $0.084(2.43)$ \\
\hline Sample 2 & 0.960 & 0.186 & 0.040 & $0.941(2.04)$ & $0.197(2.84)$ & $0.038(4.21)$ \\
\hline Sample 3 & 1.920 & 0.093 & 0.160 & $1.843(3.12)$ & $0.088(2.95)$ & $0.150(3.64)$ \\
\hline \multicolumn{7}{|l|}{ Mebendazole } \\
\hline Sample 1 & 0.218 & 0.144 & 0.061 & $0.227(1.46)$ & $0.137(2.51)$ & $0.058(3.15)$ \\
\hline Sample 2 & 0.655 & 0.048 & 0.092 & $0.642(2.53)$ & $0.046(4.26)$ & $0.097(2.58)$ \\
\hline Sample 3 & 1.966 & 0.096 & 0.184 & $1.986(1.87)$ & $0.094(3.85)$ & $0.175(3.65)$ \\
\hline Vermox $100^{\circledR}$ & 100 & - & - & $97.810(2.58)$ & $1.205(3.05)$ & $1.056(4.12)$ \\
\hline Vermox $500^{\circledR}$ & 500 & - & - & $481.520(3.42)$ & $4.852(2.74)$ & $3.782(1.86)$ \\
\hline
\end{tabular}

The values are means of three determinations. Concentrations of synthetic solutions are expressed as $\mathrm{mg} / \mathrm{ml}$; nominal and found values of pharmaceuticals as mg.

dyne 7725 manual injector. The LC column was a C8 Agilent Eclipse XDB $(150 \mathrm{~mm} \times 4.6 \mathrm{~mm} \times 5 \mu \mathrm{m})$; mobile phase was $50 \%$ acetonitrile in water at a flow rate of $1 \mathrm{ml} / \mathrm{min}$ at room temperature. The injection volume was $20 \mu \mathrm{l}$.

GC analysis was performed by a HP $6890 \mathrm{~N}$ gaschromatograph equipped with a HP Mass selective detector 5973N on a methylsilicone SE-30 HP capillary column $(30 \mathrm{~m} \times 0.25 \mathrm{~mm}$ i.d., $0.25 \mu \mathrm{m}$ film thickness $)$ at the following conditions: carrier gas, Helium at a flow rate between $0.2-0.7 \mathrm{ml} / \mathrm{min}$; oven temperature programmed from 60 to $280^{\circ} \mathrm{C}$ at $20^{\circ} \mathrm{C} / \mathrm{min}$, initial time $5 \mathrm{~min}$, final time $40 \mathrm{~min}$. Injections were accomplished in split ratio $1: 60$. The ionization of the samples was performed in electron impact mode (EI, $70 \mathrm{eV}$ ). Injector and detector were maintained at 250 and $280^{\circ} \mathrm{C}$, respectively

The photodegradation test was performed, according to the ICH Guideline for photostability testing ${ }^{19)}$ by using a light cabinet Suntest Heraeus CPS + (URAI, Milan, Italy), equipped with a Xenon lamp that respects the D65 standard of ICH norms. Samples were irradiated in a wavelength range between 300 and $800 \mathrm{~nm}$, maintaining the radiant power to $350 \mathrm{~W} / \mathrm{m}^{2}$, corresponding to a light dose of $21 \mathrm{~kJ} / \mathrm{min} / \mathrm{m}^{2}$.

Reagents ALB, FEN and MEB were purchased from Sigma-Aldrich (Germany). Absolute ethanol, acetonitrile and water, all HPLC grade, were obtained from J. T. Baker (Holland). Other solvents and reagents were of the highest purity that was commercially available. Silica Gel 60, 0.040 $0.063 \mathrm{~mm}$ (Merck, Germany). Zentel 400 (SmithKline Beecham, Italy), Vermox 100 and 500 (Janssen Cilag, Italy) pharmaceuticals were obtained commercially.

Isolation of Degradation Products The main degradation products were obtained by irradiating ethanol saturated solutions of the drugs under the Xenon lamp. The degradation process was monitored by HPLC. The exposure times were prolonged until a drug residue concentration under $20 \%$ was reached. The final exposure times resulted to be nearly $8.5,40$, and $18 \mathrm{~h}$ for ALB, FEN and MEB, respectively.

The solutions were then concentrated and fractionated by liquid chromatography on silica gel. The separation was optimized using as eluent chloroform/acetic acid $(98: 2)$ for ALB and FEN samples, and chloroform/acetic acid/formic acid $(96: 2: 2)$ for MEB sample. Three main fractions from each column were isolated and characterized as the two main photoproducts and the parent drug. The fractions were filtered through a $0.45 \mu \mathrm{m}$ PTFE filter and the eluent removed by evaporation at $40{ }^{\circ} \mathrm{C}$. The solid residues were purified by crystallization in methanol. Identity of the photoproducts were confirmed by GC-MS

Photo- and Thermal-Degradation Procedures Ethanol solutions of ALB, FEN and MEB $(20 \mathrm{mg} / \mathrm{ml})$ were exposed under the Xenon arc lamp and analyzed immediately before the degradation test and therefore at 0.25 , $0.5,1,2,4,6,8,10 \mathrm{~h}$.

Thermal-degradation study on ethanol solutions of ALB, FEN, MEB $(20 \mathrm{mg} / \mathrm{ml})$ were performed in a thermostatic bath at a temperature of $50^{\circ} \mathrm{C}$ for a total of $10 \mathrm{~h}$. The solutions were analysed at sequential times as described above. The temperature of $50{ }^{\circ} \mathrm{C}$ was selected after verified that an exposure below this value produced negligible modifications for $3 \mathrm{~h}$.

The drugs in ethanol $(20 \mathrm{mg} / \mathrm{ml})$ were finally exposed to both light and thermal degradation under the same experimental conditions above detailed.

Synthetic Samples An external validation of the HPLC method was achieved over synthetic binary mixtures in different ratios, with concentration values within the ranges used for the drugs calibrations. For the assay of commercial solid dosage forms, five tablets were weighed and reduced to a fine powder. An amount corresponding to the average of one tablet was accurately weighed, stirred and made up to a volume of $25 \mathrm{ml}$ with ethanol. The suspension obtained was sonicated for ten minutes, and then filtered with a PTFE $0.45 \mu \mathrm{m}$ membrane filter. The filtrate was diluted $1: 10$ with ethanol and finally analyzed by HPLC.

\section{Conclusions}

The knowledge of the degradation process for the benzimidazole anthelmintics appears very important because its use is high in tropical nations where temperature and light are particularly elevated. Accelerated stability study under light and high temperature of the commercially most important benzimidazoles, ALB, FEN and MEB, was carried out by chromatographic techniques. Controlled irradiation tests proved to be valid to predict drug photostability when establishing drug manipulation and conservation conditions. The formation of two main photoproducts was demonstrated, formed from two sequential reactions, a first demethylation of the estheric group followed by a decarboxylation of the carbamic group. The most resistant drug was demonstrated to be fenbendazole whereas albendazole resulted the less stable structure. Light was demonstrated to be the effective cause of degradation whereas high temperature seemed not be influent. The HPLC method could be used to assay the drug formulations during manufacturing and storage. It was demonstrated robust, accurate and precise over the intended ranges for all the studied drugs and their related degradation products.

Acknowledgment This research was supported by grants from M.I.U.R (Italy).

\section{References}

1) Muttalib M. A., Khan M. U., Hag J. A., J. Trop. Med. Hyg., 84, 159160 (1981).

2) Beijersbergen van Henegouwen G. M. J., Adv. Drug Res., 29, 79-170 
(1997)

3) Albini A., Fasani E., "Drugs Photochemistry and Photostability," ed. by The Royal Society of Chemistry, Cambridge, 1998.

4) Tønnesen H. H., Int. J. Pharm., 225, 1-4 (2001).

5) Wahlgren M., Frolov I., Lett. Trans. R. Socc. Trop. Med. Hyg., 77, 422-424 (1983).

6) Shield J., Papua New Guinea Med. J., 27, 89-94 (1984).

7) Abadi K., Am. J. Trop. Med. Hyg., 34, 128-133 (1985).

8) Cook G. C., Parasital. Today, 6, 133-136 (1990).

9) Greenhill J. V., McLelland M. A., Prog. Med. Chem., 27, 51-121 (1990).

10) Wen H., New R. R. C., Craig P. S., Br. J. Clin. Pharm., 35, 565-574 (1993).

11) El Fatih I. A. K., Ahmed M. H., Salama R. B., Int. J. Pharm., 142, $251-255$ (1996)

12) Gomes A. R., Nagaraju V., J. Pharm. Biom. Anal., 26, 919-927
(2001).

13) Del-Brutto O. H., Sotelo G., Roman G. C., Clin. Infect. Dis., 17, 730-735 (1993).

14) Velık J., Baliharov V., Fink-Gremmels J., Bull S., Lamka J., Skalova L., Res. Vet. Science, 76, 95-108 (2004).

15) Al-Kurdi Z., Al-Jallad T., Badwan A., Jaber A. M. Y., Talanta, 50, 1089-1097 (1999).

16) Torrado S., Torrado S., Torrado J. J., Cadorniga R., Int. J. Pharm., 140, $247-250$ (1996).

17) Van Tonder E. C., de Villiers M. M., Handford J. S., Malan C. E. P., du Preez J. L., J. Chromatogr. A., 729, 267-272 (1996).

18) ICH, Harmonised Tripartite Guide, "Validation of Analytical Procedures: Methodology," 1996, pp. 1—8.

19) ICH, Harmonized Tripartite Guideline, "Photostability Testing of New Drug Substance and Products," Federal Register, 62, 27115-27122 (1996). 